\title{
Kontext des öffentlichen Einkaufs
}

\subsection{Definitionen}

Im Folgenden sollen nun kurz die für vorliegende Arbeit wesentlichen Begriffe definiert werden, insbesondere Public Procurement/öffentlicher Einkauf in Abgrenzung zu Beschaffung und Vergabe, E-Government und Open Government. Zudem gilt es die jeweiligen Definitionen der Schnittmengen (siehe Abbildung 5.1) herzuleiten. Definitionen mit Relevanz für spätere Kapitel werden dort erläutert.

\subsection{1 Öffentlicher Einkauf in Abgrenzung zu Vergabe und Beschaffung}

Im Zentrum vorliegender Arbeit steht der öffentliche Einkauf. Synonym zu diesem Begriff werden in der Literatur „öffentliche Beschaffung“ und „öffentliche Vergabe“ verwendet. Dies bedarf einer Konkretisierung. In der vorliegenden Arbeit soll die Definition von Christmann, Huland und Meißner (Christmann et al., 2004, S. 5 f.) in Kombination mit der von Eßig (Eßig, 2008, S. 295) verwendet werden. Demnach ist der Einkauf in öffentlichen Verwaltungen der Oberbegriff für die Versorgung der Bedarfsträger mit Lieferungen und Leistungen. Der öffentliche Einkauf ist dadurch charakterisiert, dass der Käufer aus dem öffentlichen Sektor kommt beziehungsweise Güter und Dienstleistungen für den öffentlichen Bedarf einkauft. Dabei bezieht sich „Einkauf“ sowohl auf die Organisationseinheit als auch auf die Funktion (das Einkaufen selbst). In die 


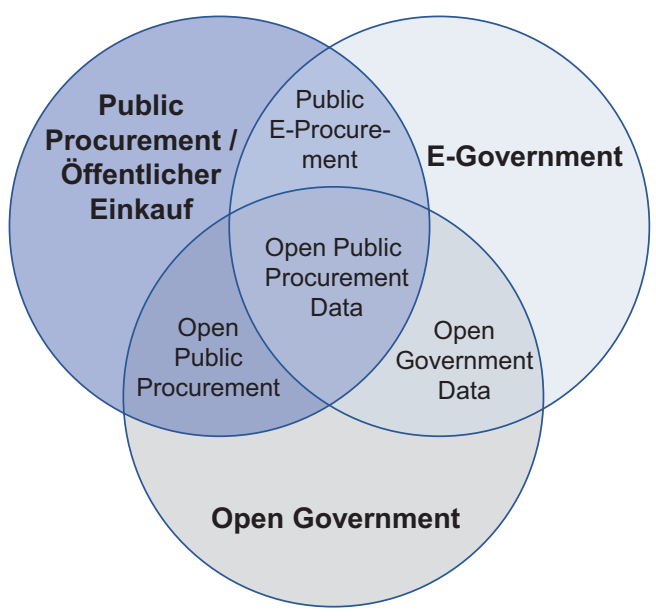

Abbildung 5.1 Begriffliche Abgrenzungen und Überschneidungen

Zuständigkeit des Einkaufs fällt die Koordination von Vergabe und Beschaffung. In dieser Arbeit wird übergreifend vom „öffentlichen Einkauf“ gesprochen.

Der Begriff der Vergabe verweist auf die Auftragsvergabe und den prozessualen Ablauf gemäß den Vergabeordnungen und dem Vergaberecht. Wichtig ist, dass die Vergabe nur einen Teil eines übergeordneten und weiterreichenden Einkaufsprozesses darstellt (siehe Abschnitt 6.3.2). In diesem Zusammenhang taucht auch der Begriff eVergabe auf. Dieser bezieht sich auf die elektronische Abwicklung von Ausschreibungsprozessen (Broens et al., 2012, S. 205). Die Nutzung der elektronischen Abwicklung ist mittlerweile im Vergaberecht vorgeschrieben. ${ }^{1}$

Beschaffung umfasst die operative Bestellabwicklung, das heißt den Prozess mit allen Transaktionen von der Bestellanforderung über den Wareneingang bis zur Rechnungsabwicklung (Christmann et al., 2004, S. 5 f.).

\subsubsection{E-Government}

In der Literatur existieren zu „E-Government“ oder ,elektronischem Regierungsund Verwaltungshandeln“" zahlreiche Definitionen. Maßgeblich für diese Arbeit ist die ,Speyerer Definition“" von E-Government:

\footnotetext{
${ }^{1}$ Mit der eVergabe wird sich Abschnitt 6.2.1.10 noch näher befassen.
} 
„Unter Electronic Government verstehen wir die Abwicklung geschäftlicher Prozesse im Zusammenhang mit Regieren und Verwalten (Government) mit Hilfe von Informations- und Kommunikationstechniken über elektronische Medien. [...] Diese Definition umfasst sowohl die lokale oder kommunale Ebene, die regionale oder Landesebene, die nationale oder Bundesebene sowie die supranationale und globale Ebene. Eingeschlossen ist somit der gesamte öffentliche Sektor, bestehend aus Legislative, Exekutive und Jurisdiktion sowie öffentlichen Unternehmen“ (von Lucke \& Reinermann, 2000, S. 1).

In dieser Definition wird vor allem die ebenenübergreifende, verwaltungsinterne Optimierung der Prozesse des öffentlichen Sektors mithilfe von Technologie betont. Damit steht E-Government für das Leitbild der Verwaltungsmodernisierung und des Bürokratieabbaus durch Prozessoptimierung mithilfe der IKT. Manuelle, papiergebundene Arbeitsschritte werden durch elektronische Transaktionen ersetzt. Die Idee, dass Bürger sich aktiv in die Gestaltung des Staats mit einbringen oder gar eine Vernetzung mit Dritten (außerhalb der Verwaltung) erfolgt, wird ebenfalls mit der Leitidee des Open Governments verbunden.

\subsubsection{Public E-Procurement}

Die Europäische Union (EU) versteht unter Public E-Procurement den

„... von der Bekanntmachung bis zur Bezahlung erfolgende[n] Einsatz elektronischer Verfahren für Kommunikation und Vorgangsbearbeitung durch Einrichtungen des öffentlichen Sektors beim Einkauf von Waren und Dienstleistungen oder der Ausschreibung öffentlicher Arbeiten“" (Europäische Kommission, 2013, S. 2).

Demnach hat die EU ein recht breites Prozessverständnis: Sie betont den Einsatz von Technologien über den Gesamtprozess von der Bekanntmachung bis zur Bezahlung.

\subsubsection{Open Government}

Unter „Open Government“ oder „offenes Regierungs- und Verwaltungshandeln“ werden sowohl technische wie auch gesellschaftspolitische Entwicklungen gebündelt, die im Kern auf die Öffnung der Politik und Verwaltung für Bürger, Gesellschaft, Wirtschaft und Wissenschaft, Transparenz, Bürgerbeteiligung und Zusammenarbeit abzielen (Groß et al., 2014, S. 6). Im Vordergrund steht die Stärkung des öffentlichen Vertrauens in die Verwaltung, ihre Effizienz und 
Effektivität sowie die Demokratie als Ganzes. Dies soll durch die Umsetzung der drei Grundprinzipien Transparenz, Partizipation und Kollaboration erreicht werden, auf die sich auch Barack Obama am 21. Januar 2009 bezog (Obama, 2009). ${ }^{2}$ Hilgers definiert:

„Unter Open Government wird die systematische Öffnung von Staat und Verwaltung im Sinne einer Entgrenzung bisheriger Organisations- und Entscheidungsstrukturen verstanden. Ziel dieser Bestrebungen ist es, unter intensiver Nutzung von Internettechnologien, mehr Transparenz, mehr Teilhabe und eine intensivere Zusammenarbeit mit den verschiedenen Anspruchsgruppen des öffentlichen Sektors zu erreichen. Über Digitalisierung, Vernetzung und Konzepte des E-Government hinausgehend ist Open Government inzwischen in zahlreichen Ländern zum praktischen Leitbild und eigenständigen Reformziel für die Modernisierung von Politik und Gesellschaft geworden“ (Hilgers, 2012, S. 631).

Hilgers baut auf der Definition Obamas auf und grenzt Open Government von E-Government ab. Während E-Government die Einführung und Verbreitung von IKT und damit die Prozessoptimierung im Rahmen der Ablauforganisation zum Ziel hat, stellt Open Government die übergreifende Vernetzung der unterschiedlichen Akteure in den Mittelpunkt und damit die Ausweitung auf Dritte außerhalb der Verwaltung (Hilgers, 2012, S. 631; Klessmann et al., 2012, S. 1). Auch Geiger und von Lucke greifen diesen Gedanken auf, indem sie Open Government als Paradigma basierend auf offenen Daten und E-Government als „Werkzeugkasten“ des Open Governments bezeichnen (Geiger \& von Lucke, 2012, S. 266). Obwohl bei E-Government das Ziel ebenfalls eine verbesserte Interaktion mit den verschiedenen Akteuren wie Bürgern und Unternehmen ist, steht dort eher ein von oben nach unten verbreiteter Ansatz sowie die Schaffung der technischen Grundlage im Vordergrund - nicht jedoch die Vernetzung und aktive Einbringung durch die Akteure von unten nach oben sowie in die Breite (zum Beispiel Wirtschaft, Wissenschaft, Gesellschaft). Open Government geht folglich über das Ziel der Bürgerorientierung im Verwaltungshandeln hinaus, indem es den liberalen Ansatz einer Bürgergesellschaft betont (von Lucke \& Große, 2009).

Von Lucke geht in seinem Verständnis von Open Government noch weiter und konkretisiert die Öffnung des gesamten informatischen Ökosystems:

„Open Government als simple Öffnung von Regierung und Verwaltung zusammenzufassen greift zu kurz. Vielmehr wird Open Government als ein Sammelbegriff für eine ganze Reihe unterschiedlicher Konzepte und Visionen verwendet, die sich mit

${ }^{2}$ Auf diese Grundprinzipien wird in Abschnitt 6.5 noch näher eingegangen. 
bestimmten Facetten einer Öffnung von Staat und Verwaltung auseinandersetzen. Hierzu zählen Überlegungen zu Transparenz 2.0, Partizipation 2.0 und Kollaboration 2.0, der Ansatz offener Innovationen, die Öffnung der Gesellschaft, die offene Gesellschaft, Überlegungen zu freien Daten sowie offene Standards, offene Schnittstellen, quelloffene Software und offene Kommunikationssysteme“ (von Lucke, 2012b, S. 3).

Es kann festgehalten werden, dass Open Government ein breites Konzept, Leitbild beziehungsweise Paradigma eines offenen Verwaltungs- und Regierungshandelns darstellt, welches noch nicht einheitlich definiert oder auf einen bestimmten Ansatz reduziert werden kann. E-Government kann als technologischer Wegbereiter angesehen werden - eine weiterführende Diskussion von Open Government fußt jedoch auf einer Auseinandersetzung mit offenen Daten und deren Verwendung durch unterschiedliche Akteure.

\subsubsection{Open Data}

„Open Data“ bezeichnet eine neue Kultur, Daten des öffentlichen Interesses maschinenlesbar, strukturiert und ohne Zugriffsbeschränkungen $\mathrm{zu}$ veröffentlichen. Hiermit soll ermöglicht werden, dass andere Anwendungen über Transaktionen wie Suchen und Filtern die Daten auffinden und weiterverarbeiten können. Die Open Knowledge Foundation (OKFN) Deutschland definiert:

„Offene Daten sind Daten, die von jedermann frei verwendet, nachgenutzt und verbreitet werden können maximal eingeschränkt durch Pflichten zur Quellennennung und ,sharealike“" (Open Knowledge Foundation Deutschland e. V., 2015).

In diesem Kontext geht es um Daten, auf die eine juristische oder natürliche Person keinen alleinigen Anspruch erheben kann. Die Veröffentlichung kann durch die Wissenschaft, die Wirtschaft, NGOs, Bürger, aber auch durch die öffentliche Hand selbst erfolgen. Explizit ausgenommen sind personenbezogene Daten, Daten, deren Öffnung ein Sicherheitsrisiko darstellen, Geschäfts- und Betriebsgeheimnisse sowie Verstöße gegen das Urheberrecht (Geiger \& von Lucke, 2012, S. 269; Groß et al., 2014, S. 7).

Bei der Öffnung der Daten gilt es zu berücksichtigen, dass es zwischen vollständiger Öffnung von Daten beziehungsweise vollständiger Nicht-Öffnung mehrere Zwischenstufen geben kann. Es handelt sich also nicht um einen festdefinierten Zustand, wie Abbildung 5.2 anhand der vier Kriterien Zugang, Maschinenlesbarkeit, Nutzungskosten, Lizenzierung und Rechte verdeutlicht: 


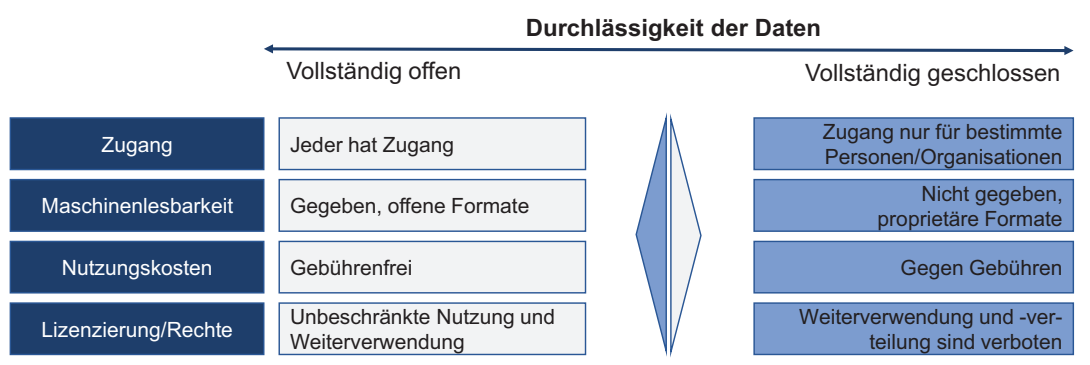

Abbildung 5.2 Durchlässigkeit von Daten (in Anlehnung an Chui et al., 2014, S. 6, übersetzt durch Verfasserin)

Bei Öffnung etwaiger Datenbestände ist eine Abwägung vonnöten, welcher Grad der Offenheit gewünscht und sinnvoll ist, auch unter Beachtung der Interessenlage (beispielsweise zwischen vollständiger Offenlegung gewünscht von den Steuerzahlern einerseits und Wahrung von Geschäftsgeheimnissen eingefordert durch Unternehmen oder Schutz des Amtsgeheimnisses bevorzugt von den Verwaltungen andererseits) (Klessmann et al., 2012, S. 38; von Lucke, 2012b, S. 20; von Lucke \& Große, 2009, S. 2 f.).

\subsubsection{Open Government Data}

Im Kontext von Daten der öffentlichen Hand wird von „Open Government Data“ gesprochen; sie sind ein Teil aller offenen Datenbestände, die der interessierten Allgemeinheit ohne Einschränkung frei zugänglich gemacht werden sollen, beispielsweise (Ubaldi, 2013, S. 6):

- Geschäftsinformationen (inklusive Informationen der Industrie- und Handelskammern)

- Register, Patente und Informationen zu Handelsmarken sowie öffentliche Ausschreibungsdatenbanken beziehungsweise Verträge

- Geografische Informationen (inklusive Informationen zu Adressen, Luftbildaufnahmen, Gebäuden, Katasterinformationen, geodätischen Netzwerken, Geologie, hydrografischen Daten und topografische Informationen)

- Rechtsauskünfte (inklusive Entscheidungen der nationalen, ausländischen und internationalen Gerichtshöfe, nationale Gesetzgebung und getroffene Übereinkommen) 
- Meteorologische Informationen (inklusive Klimadaten, Modellrechnungen sowie Wettervorhersagen)

- Soziale Daten (inklusive diverser Statistiken zur Wirtschaft, Beschäftigung, Gesundheit, Bevölkerung und Verwaltung)

- Transportinformationen (inklusive Informationen zu Verkehrsstörungen, Baustellen, öffentlichem Verkehr und Kfz-Registrierung).

Es werden weitestgehend übereinstimmend die zehn Prinzipien der Sunlight Foundation $\mathrm{zu}$ offenen Regierungsinformationen angewendet, um Offenheit bewerten zu können. Diese Prinzipien sind: Vollständigkeit, aus der Primärquelle stammend, Aktualität, einfacher physischer und elektronischer Zugang, Maschinenlesbarkeit, Diskriminierungsfreiheit, Verwendung offener Standards, Lizenzfreiheit, Dauerhaftigkeit und angemessene Nutzungskosten (Sunlight Foundation, 2010).

\subsubsection{Open Public Procurement (Data)}

„Open Public Procurement“ oder „offener öffentlicher Einkauf“ sind Begriffe, die auf einen Teilbereich von Open Government verweisen. Einkauf wird hier im Sinne des Gesamtprozesses von der Planung bis zur Ausführung verstanden. Ein offen angelegter Einkauf informiert nicht nur über den erfolgreichen Bieter als Teil der Vergabe, sondern auch über andere Bestandteile des Prozesses. „Open Public Procurement Data“ oder „Offene öffentliche Einkaufsdaten“ bezeichnen wiederum einen Teilbereich von Open Government Data. Hierbei geht es um die frei zugängliche Bereitstellung öffentlicher Einkaufsdaten entlang des gesamten Beschaffungszyklus von der Bedarfsermittlung bis zur Rechnungsprüfung (Open Contracting Partnership, 2013, S. 4).

Im Folgenden können die politischen Positionen zur Öffnung des öffentlichen Einkaufs vorgestellt werden, die auf die hier geklärten Begriffe zurückgreifen.

\subsection{Politische Positionen zur Öffnung des öffentlichen Einkaufs}

Diverse internationale und nationale Strategien und Programme greifen Themen wie Transparenz, Antikorruption, offene Regierungsdaten sowie den öffentlichen Einkauf auf. Sie werden nun hinsichtlich ihres Bezugs zur Öffnung 
des öffentlichen Einkaufs skizziert. Aus internationaler Sicht wird die G203 beleuchtet, anschließend die $\mathrm{G}^{4}$ und die EU. Dann rückt Deutschland auf Bundesebene in den Fokus. Im Anhang findet sich eine Tabelle, die eine Gegenüberstellung der vorgestellten Regierungsprogramme und ihres Bezugs zum offenen öffentlichen Einkauf leistet (Tabelle A.2).

„Vor allem Nichtregierungsorganisationen und Journalist(innen) wollen den Vorsprung des Staates vor der Bevölkerung verkleinern“ (Semsrott, 2016, S. 7). Zwischenstaatliche Organisationen, also Intergovernmental Organizations (IGOs), und NGOs sind Treiber dieses Diskurses, indem sie beispielsweise Transparenz bei öffentlichen Ausschreibungsverfahren verlangen, Verträge überprüfen und das Wirken alternativer Vermögensverwaltungsfonds offenlegen (Morozow \& Bria, 2017, S. 53). Die Sicht auf Positionen der IGOs beziehungsweise NGOs schließt sich daher an und wird ebenfalls mit einer tabellarischen Gegenüberstellung beendet (siehe Tabelle A.3 im Anhang), bevor die Ziele des öffentlichen Einkaufs und von Open Public Procurement möglichst präzise gefasst und illustriert werden. Wirtschaft, Wissenschaft und Medien als Akteure, die eher eine begleitende und unterstützende denn eine treibende Funktion wahrnehmen, ergänzen die Ausführungen.

\subsubsection{Internationale und nationale Strategien und Programme}

\subsubsection{Der G20-Antikorruptionsplan 2019 bis 2021}

Gemäß G20-Antikorruptions-Plan 2019 bis 2021 ist es die Priorität der G20, Korruption vorzubeugen und zu bekämpfen. Der Aktionsplan sieht zwei wesentliche Abschnitte (A und B) vor, in Abschnitt B (,Develop further targeted actions where the G20 can best add value") finden sich unter dem ersten

\footnotetext{
${ }^{3}$ Die G20 ist ein informeller Zusammenschluss aus 19 Staaten und der EU, welcher seit 1999 besteht. Die G20 bietet ein Forum zum Austausch globaler Themen an. Zu ihr zählen Argentinien, Australien, Brasilien, Deutschland, die EU, Frankreich, Indien, Indonesien, Italien, Japan, Kanada, Mexiko, Russland, Saudi-Arabien, Südafrika, Südkorea, Türkei, das Vereinigte Königreich, die Vereinigten Staaten, die Volksrepublik China (Presse- und Informationsamt der Bundesregierung, 2019).

${ }^{4}$ Die G8 ist ein informeller Zusammenschluss der wirtschaftsstärksten Länder der westlichen Welt. Auch hier ist das Ziel eine Zusammenkunft zur Erörterung weltpolitischer Themen. Seit 25. März 2014 zählt Russland aufgrund der Krimkrise nicht mehr zur G8 - seit diesem Zeitpunkt operiert das Gremium wieder als G7. Die G7-Staaten bestehen aus den USA, Deutschland, Japan, Frankreich, Italien, Großbritannien und Kanada (Presseund Informationsamt der Bundesregierung, 2019).
} 
Absatz „Stärkung und Förderung der Integrität und Transparenz im öffentlichen und privaten Sektor" die Absichtserklärungen, die Nutzung offener Daten zu fördern, Korruptionsrisiken im öffentlichen Einkauf, dem Haushalt sowie den Steuerbehörden zu identifizieren und zu artikulieren, die öffentlichen Institutionen zu ermutigen, Antikorruptionsinitiativen zu implementieren und weiterhin Wege zu nutzen, um die gerichtliche Integrität zu schützen und zu stärken sowie AntiKorruptionsmaßnahmen in besonders anfälligen Sektoren wie zum Beispiel dem Infrastruktur-/Bausektor zu vertiefen (G20, 2010, S. 4; OECD, 2018a, S. 5).

\subsubsection{Die Open Data-Charter der G8}

Im Juni 2013 wurde die Open Data-Charter durch die G8-Staaten unterzeichnet. Die Unterzeichnerländer stimmen darin überein, dass offene Daten eine noch unerschlossene Ressource mit großem Potential sind, mit denen besser miteinander vernetzte Gesellschaften herausgebildet werden können, die die Bedürfnisse der Bürger besser adressieren und Innovationen und Wohlstand unterstützen. Jeder G8-Staat verpflichtet sich, einen eigenen nationalen Aktionsplan zu veröffentlichen, der niederlegt, wie die Open Data-Charter gemäß den gegebenen nationalen Rahmenbedingungen umgesetzt wird. Zudem ist jährlich ein Bericht über Fortschritte zu verfassen. Die fünf Grundprinzipien der Charter umfassen: Offene Daten als Standard; Qualität und Quantität; Nutzbarkeit für alle; Veröffentlichung von Daten für ein verbessertes Regierungshandeln und Veröffentlichung von Daten für Innovationen (Bundesministerium des Innern, 2014b, S. 4). Die Open Data-Charter benennt 14 Datenkategorien: von Unternehmen, Verbrechen und Justiz, Erdbeobachtung, Erziehung, Energie und Umwelt über Finanzen und Verträge, Geodaten, globale Entwicklung, Regierungsverantwortung und Demokratie, Gesundheit, Wissenschaft und Forschung bis zu Statistik, sozialer Mobilität und Gemeinwohl sowie Transport und Infrastruktur. Offene öffentliche Einkaufsdaten werden explizit unter dem Punkt „Finanzen und Verträge" erwähnt und spezifiziert: Transaktionsvolumina, vergebene Verträge, Ausschreibung, zukünftige Ausschreibungen sowie lokales und nationales Budget (geplant und verausgabt) sollen veröffentlicht werden (G8, 2013). Erstmals werden offene Daten und offene öffentliche Einkaufsdaten hier klar adressiert.

\subsubsection{Europäische Strategien und Programme}

\subsubsection{Digitaler Binnenmarkt}

Am 06. Mai 2015 wurde die Strategie für einen digitalen Binnenmarkt für Europa durch die Europäische Kommission vorgestellt (Europäische Kommission, 2015). Der „Digitale Binnenmarkt“ ist Bestandteil und Weiterentwicklung der 
Strategie „Digitale Agenda für Europa“, die im Mai 2010 verabschiedet wurde. Die Digitale Agenda für Europa unter dem Dachprogramm „Strategie Europa 2020“ soll dazu beitragen, dass Europas Bürger und Unternehmen von den digitalen Technologien maximal profitieren (Europäische Kommission, 2010a; b). Die Strategie für einen digitalen Binnenmarkt umfasst unter anderem den Aufbau eines europäischen Datenraums (Europäische Kommission, 2017; European Commission, 2019a).

Hauptziel ist die Erschließung des bestmöglichen Nutzens digitaler Daten, um so einen Mehrwert für die Wirtschaft und Gesellschaft zu erzeugen. Zu dem Zweck soll die Wiederverwendbarkeit verschiedenster Daten und ihr freier Verkehr ermöglicht werden. Ein gemeinsamer Datenraum soll als Treiber von Wachstum und Innovationen der Entwicklung neuer Produkte und Dienstleistungen basierend auf Daten den Weg bereiten. Die Strategie enthält mehrere Schwerpunkte: einen Vorschlag für die Überarbeitung der Direktive über die Weiterverwendung öffentlicher Informationen (siehe Abschnitt 7.1.1), eine Aktualisierung der Empfehlung aus 2012 bezüglich des Zugangs und der Vorhaltung wissenschaftlicher Informationen sowie einen Leitfaden für die gemeinsame Nutzung von Daten des Privatsektors (European Commission, 2019a).

Diese Ansätze sind verbunden mit der Regulierung des freien Flusses nichtpersönlicher Daten in der EU, welche sicherstellt, dass keine Barrieren - wie zum Beispiel Restriktionen bezüglich Datenlokalisierung - die Entwicklung des europäischen Datenraums beeinträchtigen werden (European Commission, 2019a). An den Ansätzen werden zwar keine expliziten Aktivitäten rund um öffentlichen Einkaufsdaten erkennbar, doch haben sie einen rahmengebenden Einfluss.

\subsubsection{Europäischer E-Government-Aktionsplan}

Für die Europäische Union wurde von der Kommission der Europäische E-Government-Aktionsplan 2016 bis 2020 als Teil der Strategie des „Digitalen Binnenmarkts für Europa“ initiiert. Er soll mittels Bündelung konkreter operativer Maßnahmen dazu beitragen, bestehende Barrieren für den digitalen Binnenmarkt $\mathrm{zu}$ reduzieren und die weitere Fragmentierung öffentlicher Stellen zu verhindern. Als Ziel wird formuliert, dass die öffentlichen Stellen der EU bis

„2020 offene, effiziente und inklusive Einrichtungen werden, die grenzübergreifende, personalisierte, nutzerfreundliche und - über alle Abläufe hinweg - vollständig digitale öffentliche Dienste für alle Menschen und Unternehmen in der EU anbieten" (Europäische Kommission, 2016, S. 2). 
Weiters wird die hierfür besondere Bedeutung der Öffnung der Daten zwischen den öffentlichen Verwaltungen sowohl in den Mitgliedsstaaten als auch über Grenzen hinweg herausgestellt. Mit Blick auf die vorliegende Arbeit ist die im ersten von insgesamt 20 Aktionspunkten enthaltene Maßnahme hervorzuheben, die Weiterentwicklung der Mitgliedsstaaten zur vollständig elektronischen Auftragsvergabe und zur Nutzung von Auftragsregistern bis 2019 unter dem Stichwort „E-Procurement“ voranzutreiben.

\subsubsection{Nationale Strategien und Programme auf Bundesebene}

\subsubsection{Digitale Verwaltung 2020}

Im Rahmen der „Digitalen Agenda 2014-2017“ wurde mit dem Handlungsfeld „Innovativer Staat“ die übergreifende Digitalisierung der Verwaltung festgeschrieben (Bundesministerium für Wirtschaft und Energie et al., 2014, S. $18 \mathrm{ff}$.).

Mit dem E-Government-Gesetz (EGovG; siehe auch Abschnitt 7.1.4), welches am 01. August 2013 in Kraft getreten ist, legte die Bundesregierung den rechtlichen Rahmen für die Ausgestaltung des E-Governments auf Bundesebene fest. Hier geht es um durchgängige, elektronisch vernetzte und medienbruchfreie Prozesse zwischen den Beteiligten (Bundesministerium des Innern, 2019b, S. 4). Anschließend beschloss das Bundeskabinett am 08. April 2014 für die 18. Legislaturperiode das Programm „Digitale Verwaltung 2020", welches die Umsetzung des E-Government-Gesetzes an der Schnittstelle zur Ausgestaltung des Handlungsfeldes „Innovative Verwaltung“ koordinieren soll.

Für die vorliegende Arbeit ist im Zuge der Umsetzung des E-GovernmentGesetzes die Erstellung eines Aktionsplans zur Implementierung der Open DataCharter relevant, zu der sich Deutschland als G8-Land 2013 bekannt hat (Bundesministerium des Innern, 2014a, S. 31 ff.) Im Aktionsplan sind Daten des öffentlichen Einkaufs nicht explizit genannt, allerdings kann der bereits geschaffene Rahmen auf sie angewendet werden (Bundesministerium des Innern, 2017a, S. $78 \mathrm{ff}$.).

Unter Einzelprojekten des Bundes wird die E-Beschaffung als Maßnahme beim Beschaffungsamt genannt:

„Mit dem neu zu initiierenden Projekt E-Beschaffung sollen wichtige Prozesse bei Beschaffungen des Bundes standardisiert und digitalisiert werden. Die Beschaffung der Ressorts soll soweit möglich interoperabel gestaltet werden. Dies geschieht aus 
Gründen der Generierung von Synergieeffekten auf Basis der bereits bei einigen Ressorts bestehenden standardisierten und digitalisierten Verfahren. In diesem Kontext wird auf das Europäische Vergaberecht verwiesen, das auch Barrierefreiheit ausdrücklich berücksichtigt" (Bundesministerium des Innern, 2014a, S. 35 ff.).

So sollte ab 2014 der Nutzungsgrad und das Angebot des Kaufhauses des Bundes $^{5}$ erweitert, anschließend in 2014 und 2015 die $^{x V^{2} r g a b e}{ }^{6}$ fokussiert werden. Bis Ende 2015 sollte der Ausbau des Vergabeportals und die Einrichtung eines europaweit einheitlichen Zugriffs für die Bieter abgeschlossen sein. Dies betrifft jedoch nur die Vergabestellen des Bundes; auf eine mögliche Teilnahme der Länder und Kommunen wird hingewiesen. Bis Ende 2017 sollte die Entwicklung eines standardisierten elektronischen Vergabemanagementsystems abgeschlossen sein und folgend bis 2018 die medienbruchfreie Nutzung der E-Rechnung. Hier konnten auf Bundesebene Fortschritte erzielt werden. Offene Einkaufsdaten waren und sind hier aber ebenfalls nicht im Fokus (Bundesministerium des Innern, 2017a, S. 88 ff.).

\subsubsection{Nationale E-Government-Strategie}

Der IT-Planungsrat koordiniert die Zusammenarbeit und Projekte von Bund und Ländern in allen Fragen der IKT in ihrer Anwendung auf den öffentlichen Sektor. Er beschloss am 24. September 2010 eine Nationale E-Government-Strategie (NEGS) bis 2015 (IT-Planungsrat, 2010). Die NEGS ist eine ebenenübergreifend erarbeitete Leitlinie, um E-Government und die IKT der Verwaltungen gemeinsam weiterzuentwickeln. Sie wurde, basierend auf den Erfahrungen von 2010 bis 2015, ab 2015 fortgeschrieben und richtet sich auf fünf Ziele: An erster Stelle wird der Nutzen für Bürger, Unternehmen und die Verwaltung genannt, dann Wirtschaftlichkeit, Effizienz und Leistungsfähigkeit, an dritter Stelle Informationssicherheit und Datenschutz, an vierter Stelle Transparenz und gesellschaftliche Teilhabe und an fünfter Stelle Zukunftsfähigkeit und Nachhaltigkeit (IT Planungsrat, 2015).

\footnotetext{
${ }^{5}$ Kaufhaus des Bundes: Das Kaufhaus des Bundes ist eine elektronische Einkaufsplattform für den Bund und seine zugehörigen Behörden und Einrichtungen. Dabei bündeln vier zentrale Vergabestellen ihre Bedarfe und schließen Rahmenverträge mit der Privatwirtschaft ab (Geschäftsstelle Kaufhaus des Bundes, 2019).

${ }^{6} \mathrm{x}$ Vergabe: Dies ist eine plattformübergreifende Schnittstelle, die den Zugang von Bietern $\mathrm{zu}$ unterschiedlichen Vergabeplattformen standardisieren und vereinfachen soll (Karich, 2017). Siehe dazu ausführlicher Abschnitt 6.4.2.
} 
Für jedes der fünf Teilziele wurden 16 Unterziele festgelegt. Unter dem vierten Teilziel „Transparenz und gesellschaftliche Teilhabe“ findet sich mit dem zwölften Unterziel „Open Data und Informationsfreiheit werden gefördert“ der Hinweis auf offene Daten (IT Planungsrat, 2015, S. 15). Im Kontext der besseren Nachvollziehbarkeit staatlichen Handelns sowie Transparenz wird erläutert, dass

„... offene Informationen aus Politik und Verwaltung, [...] diskriminierungsfrei, adressatengerecht und benutzerfreundlich für die jeweiligen Zielgruppen - unter Beachtung von Datenschutz- und Sicherheitsaspekten - in standardisierten und maschinenlesbaren Formaten zur Verfügung gestellt werden (sollen)“ (IT Planungsrat, 2015, S. 15).

In der nationalen E-Government-Strategie ist damit die optimierte Bereitstellung offener Daten enthalten; die Konkretisierung der Datenbestände oder Benennung offener öffentlicher Einkaufsdaten fehlen jedoch.

\subsubsection{Ausgewählte IGOs und NGOs}

\subsubsection{Weltbank}

Im Zentrum der Arbeit der Weltbank, einer Sonderorganisation der Vereinten Nationen, steht die Vergabe von zinsgünstigen, projektbezogenen Krediten an Entwicklungsländer mit Blick auf Bildung und Gesundheit, Landwirtschaft und ländliche Entwicklung, Umweltschutz, Infrastruktur, große industrielle Bauprojekte und Regierungsführung (Governance) (zum Beispiel Antikorruption, Entwicklung von Rechtssystemen/-institutionen). Über diese Kredite sollen private und ausländische Direktinvestitionen gefördert werden (The World Bank Group, 2019f). ${ }^{7}$ Daher muss sich die Weltbank mit dem Thema „öffentlicher Einkauf" ebenso befassen wie mit Fragen der Transparenz und Korruption. Es muss ihr ein Hauptanliegen sein, den öffentlichen Einkauf in einer bestimmten Art und Weise abzuwickeln. Angesichts der Größe und Bedeutung der Institution erscheint ein Blick auf ihre Haltung lohnenswert.

\footnotetext{
${ }^{7}$ Sie vergab 2017 etwa 62 Milliarden US-Dollar an Krediten, Zuschüssen, Beteiligungsinvestitionen und Garantien an Entwicklungs- und Transitionsländer (The World Bank Group, 2017b, S. 21). Die Finanzierung erfolgt durch die Mitgliedsstaaten. Beitragshöhe sowie Stimmrechtsanteil richten sich auch hier nach der Wirtschaftskraft. Weiters dienen die weltweiten Kapitalmärkte der Finanzierung (The World Bank Group, 2019e).
} 
Mit Blick auf den öffentlichen Einkauf versucht die Weltbank in ihrer Doppelrolle als Förderer der Effizienz in den Ländern ihrer Kunden sowie als (Projekt-) Auftraggeber zwei Ziele zu erreichen: Nach außen gerichtet versucht sie, den öffentlichen Einkauf aufgrund seiner Bedeutung für die Wirksamkeit öffentlicher Ausgaben in den Ländern ihrer Kunden zu optimieren. Nach innen gerichtet versucht sie, genau dies zur Erreichung eigener Ziele wie Wettbewerb, Ökonomie, Effizienz und Transparenz in eigenen Projekten umzusetzen (Independent Evaluation Group, 2014, S. 1).

Folgende Schwerpunkte artikuliert die Weltbank, um die strategische Rolle des Einkaufs zu unterstützen: Verbesserung der Effizienz im öffentlichen Einkauf, sodass mehr Gelder in andere öffentliche Basisdienste wie zum Beispiel Gesundheit, Bildung, Infrastruktur investiert werden können; Modernisierung des Einkaufs, um eine bessere Passfähigkeit, Auswahl, Qualität und einen höheren Mehrwert zu erreichen; bessere nationale Einkaufssysteme, welche nachhaltige Entwicklungsziele unterstützen; Erhöhung der Transparenz im öffentlichen Einkauf durch Nutzung der IKT (The World Bank Group, 2019c). Die Ziele sind zusammengefasst Wirtschaftlichkeit und Effizienz kombiniert mit nachhaltiger Entwicklung.

Auf der Webseite finden sich im „Open Knowledge Repository“ unter dem Suchbegriff ,public procurement" mit weltweitem Bezug etwa 1400 Einträge (Zeitraum 2010 bis 2019, Stichwort „Procurement“, Stand Oktober 2019). Hiervon entfallen etwa $80 \%$ auf den nicht-europäischen/nicht-asiatischen Raum; die restlichen $20 \%$ beziehen sich im Wesentlichen auf Osteuropa. Dies spiegelt den Fokus der Weltbank auf Entwicklungs- beziehungsweise Schwellenländer wider. Zum Thema Open Government Data finden sich etwa 950 Einträge, die Verteilung ist vergleichbar mit der oben genannten. An der Schnittstelle zwischen öffentlichem Sektor, Effizienz, Benchmarking, Antikorruption, Transparenz, Vertragsmanagement sowie Open Government finden sich zahlreiche Publikationen, die jedoch keinen Schwerpunkt zu Open Public Procurement oder Open Contracting herausbilden (The World Bank Group, 2019b). Von 2012 bis 2014 war die Weltbank Gastgeber der OCP (siehe Abschnitt 5.2.4.6 Open Contracting Partnership). Im Blog auf der Website finden sich ein bis zwei Referenzartikel pro Jahr zum Themengebiet (The World Bank Group, 2019a). Demnach scheint die Weltbank Open Public Procurement aktuell keine hohe Bedeutung zuzuweisen. 


\subsubsection{OECD}

Die Organisation für wirtschaftliche Zusammenarbeit und Entwicklung (Organisation for Economic Co-operation and Development, OECD) ist eine internationale Organisation mit 36 Mitgliedsstaaten und etwa 2500 Mitarbeitern, die sich der Demokratie und Marktwirtschaft verpflichtet fühlt (OECD, 2019d). ${ }^{8}$

Die OECD bietet im Arbeitsgebiet „Public Governance“ unter dem Themenschwerpunkt „Public Procurement“ umfangreiches Material. Sie sieht drei Entwicklungsrichtungen: erstens die Gewährleistung von Basisdiensten wie Gesundheit, Infrastruktur und Energie, zweitens die Sicherstellung des Mehrwerts durch Effizienz und Integrität und drittens das Erreichen sozialer, innovativer und umweltschonender Ambitionen. Unter dem zweiten, hier relevanten Thema unterscheidet die OECD nochmals zwischen Bewertung und Effizienz einerseits und Integrität andererseits. Unter Effizienz finden sich überwiegend Ansätze zur Bewertung, zum Vergleich und zur Verbesserung des gesamten Einkaufszyklus und der Systeme sowie zur Bündelung der Einkaufsbedarfe über Leistungsindikatoren.

Unter dem Aspekt der Integrität wird betont, dass diese in allen Phasen des Einkaufsprozesses zum Tragen komme und es meist multilaterale Mechanismen seien, die auf eine Gesamtverbesserung hinwirken. Insgesamt könnten Transparenz, die Einbindung von Stakeholdern, Zugang, E-Procurement sowie Steuerung und Kontrolle zu einer höheren Integrität im öffentlichen Einkauf beitragen (OECD, 2019b). Unter „Digital Governance“ finden sich die Themen Open Government und Open Data. Hier wurde zum Beispiel der Open Government Data Report veröffentlicht, der ebenfalls auf die Bedeutung von Open Contracting und die Nutzung des Open Contracting Data Standard (OCDS) verweist (OECD, 2018b, S. 184 ff.).

Die OECD arbeitet eng mit der Open Government Partnership (OGP) und der OCP zusammen, unter anderem auch im Rahmen der Contracting Five Partnership (OECD, 2019c). Hierzu an späterer Stelle mehr, zunächst jedoch ein kurzer Blick auf eine weitere wichtige NGO.

${ }^{8}$ Die Organisation finanziert sich aus Beiträgen der Mitgliedsstaaten, also aus Steuermitteln. Das Zentralbudget (2017: 374 Millionen Euro) wird nach einem von der Wirtschaftskraft abhängigen Beitragsschlüssel von den Mitgliedern getragen. Mit 20,6\% waren die USA 2017 der größte Beitragszahler, gefolgt von Japan $(9,4 \%)$ und Deutschland $(7,4 \%)$ (OECD, 2019a). 


\subsubsection{Transparency International}

Transparency International (TI) ist eine internationale NGO, die 1993 in Den Haag ins Leben gerufen wurde. Im gleichen Jahr erfolgte die Gründung von Transparency International e. V. mit Sitz in Berlin als gemeinnütziger Verein. Ziel ist die weltweite Reduktion von Korruption sowie die Prävention von Straftaten, die mit Korruption im Zusammenhang stehen (Transparency International e. V., 2019e). TI ist ein Dachverband; Mitglieder des Vereins sind mehr als 100 nationale Vertretungen („National Chapter"), Organisationen auf nationaler Ebene sowie Einzelpersonen, die sich in ihren Heimatländern der Korruptionsbekämpfung widmen (Transparency International e. V., 2019d). Die Schwerpunkte der Arbeit umfassen politische Korruption, Korruption bei öffentlichen Ausschreibungen, Korruption im Privatsektor, internationale Konventionen gegen Korruption sowie Armut und Entwicklung. ${ }^{9}$

Mit Blick auf offene Verträge arbeitet TI International eng mit der OGP zusammen. Beispielsweise wurden Empfehlungen zur Gestaltung nationaler Aktionspläne zusammengefasst, die die Forderungen der Initiative „Open Contracting Partnership (OCP)“ aufgreifen. So soll erstens der OCDS genutzt werden, um maschinenlesbare Daten über den gesamten Einkaufsprozess zu veröffentlichen, zweitens sollten alle Verträge „open by default“ sein, um eine Kultur sozialer Innovationen und den Wettbewerb zu fördern, und drittens sollen die Entwicklung und Implementation von Mechanismen für Konsultationen sowie ein unabhängiges Monitoring durch die Bürger und die Zivilgesellschaft unterstützt werden.

TI Deutschland e.V. agiert mit einem jährlichen Budget von etwa 0,5 Millionen Euro. Die Organisation engagiert sich mit Blick auf den öffentlichen Einkauf unter dem Stichwort „Vergabe“ mit einer eigenen Arbeitsgruppe, deren Kernforderungen lauten (Transparency International Deutschland e. V., 2019b):

${ }^{9}$ Die Finanzierung erfolgt durch verschiedene Quellen, hauptsächlich durch staatliche Organe und internationale Organisationen (etwa $75 \%$ ), aber auch durch Stiftungen und Privatpersonen. 2017 zählten zu den größten Spendern des jährlichen Budgets von etwa 22 Millionen Euro unter anderem die Europäische Kommission, das Department of Foreign Affairs, Trade and Development (DFATD) in Kanada und das Bundesministerium für wirtschaftliche Zusammenarbeit und Entwicklung (BMZ) in Deutschland (Transparency International e. V., 2017, S. 37 ff.). Die Finanzströme werden auf der Homepage veröffentlicht und TI bemüht sich um Unabhängigkeit (Transparency International e. V., 2019f). 
1) rechtssichere und praktikable Gestaltung des Vergaberechts durch den Gesetzgeber,

2) Vermeidung der Überlagerung der Bedarfsdeckung durch vergabefremde Zwecke,

3) Definition bundeseinheitlicher Schwellenwerte zur effizienten und sicheren Abwicklung,

4) Ausschluss unzuverlässiger Unternehmen, zum Beispiel über das 2017 eingeführte Wettbewerbsregister,

5) ein leicht umsetzbarer Rechtsschutz für Bieter,

6) Informationen seitens öffentlicher Auftraggeber zum Vergaberecht, zu bestehenden Korruptionsrisiken sowie Möglichkeiten der Korruptionsreduktion sowie

7) Aufdeckung und Sanktionierung von Verstößen gegen das Vergabe- und Dienstrecht.

TI Deutschland scheint demnach eher auf traditionellere Forderungen zu setzen, überwiegend die Herstellung und Anwendung des Rechtsrahmens. Sie hat zum Thema „Open Contracting“ bislang keine offiziellen Positionen verfasst, befürwortet aber eine stärkere Transparenz im Unterschwellenbereich (Lantermann, 2015, S. 11).

\subsubsection{Open Knowledge Foundation Deutschland}

Die Open Knowledge Foundation (OKFN) International wurde als Non-profitDachorganisation 2004 mit Sitz in Großbritannien gegründet und ist mittlerweile in mehr als 46 Ländern vertreten (Open Knowledge Foundation International, 2019b). Ihr Ziel ist es, Zivilgesellschaften den Wert offener Daten aufzuzeigen und Interessierte zu unterstützen, den Zugang zu diesen Daten zu erhalten und sie nutzen zu können, um so konkrete gesellschaftliche Probleme zu lösen. Die Finanzierung erfolgt durch verschiedene Großunternehmen, Stiftungen, Regierungen, internationale Organisationen und einzelne Spender. Genauere Zahlen gibt sie allerdings nicht transparent bekannt (Open Knowledge Foundation International, 2019a).

Die OKFN Deutschland e. V. ist eine von zehn nationalen Vertretungen ${ }^{10} . \mathrm{Im}$ Jahr 2017 belief sich das zur Verfügung stehende Budget auf etwa 2 Millionen

\footnotetext{
${ }^{10}$ Außer Großbritannien und Deutschland sind dies Belgien, Brasilien, Finnland, Griechenland, Japan, Nepal, Schweden und die Schweiz (Open Knowledge Foundation International, 2019c).
} 
Euro, zu $81 \%$ bereitgestellt durch öffentliche Stellen (Open Knowledge Foundation Deutschland e. V., 2019a). Die Ziele der OKFN Deutschland sind offen zugängliches Wissen und demokratische Teilhabe, die Stärkung der digitalen Mündigkeit und des ethischen Umgangs mit Technologie und Vernetzung (Open Knowledge Foundation Deutschland e. V., 2019e). Seit ihrer Gründung im Jahr 2011 in Berlin hat sie 44 Projekte mit mittlerweile 39 Mitarbeitern und 1400 Mitgliedern umgesetzt (Open Knowledge Foundation Deutschland e. V., 2019d), unter anderem Kampagnen, Hackathons, CommunityFörderungen, Bildungsangebote und eigene Softwarelösungen.

Mit Blick auf den öffentlichen Einkauf ist insbesondere das Projekt „Digiwhist“ von besonderer Relevanz für vorliegende Arbeit. Es wurde zwischen 2015 und 2017 umgesetzt und über das EU-Programm „Horizon 2020“ finanziert. Mit „Digiwhist“ wurde das Anliegen verfolgt, sowohl das Vertrauen in die Regierungen zu steigern als auch die Effizienz der öffentlichen Vergaben in Europa zu verbessern. Zu dem Zweck wurden von 35 Nationen $^{11}$ simultan Informationen $\mathrm{zu}$ öffentlichen Vergaben oberhalb und unterhalb der nationalen Schwellenwerte sowie zur Finanz- und Eigentümerstruktur der Gewinner der Ausschreibungen systematisch gesammelt, strukturiert und analysiert. Während 20 der 28 teilnehmenden Länder auf Daten des Unterschwellenbereichs zugriffen, wurden für Deutschland lediglich Oberschwellenwerte zur Auswertung genutzt (Cosinex $\mathrm{GmbH}, 2018)^{12}$, was auf den Handlungsbedarf für den Unterschwellenbereich verweist. Die Daten hat man dann wiederum mit aggregierten Haushaltsund Einkommenserklärungen verknüpft, um so Unregelmäßigkeiten aufdecken und hinterfragen zu können. Im Kern sind die Ergebnisse auf 35 Portalen (ein zu entwickelndes Portal pro Land ${ }^{13}$ ) dargestellt und über https://opentender.eu/ in einem gemeinsamen, übergreifenden Portal abgebildet worden. Hierüber können die einzelnen Datensätze sowie Dokumente heruntergeladen, interaktive Analysewerkzeuge genutzt sowie mit den Nutzern des Portals kommuniziert werden

\footnotetext{
${ }^{11}$ Dies sind die 28 EU-Mitgliedsstaaten, die Europäische Kommission, Armenien, Georgien, Island, Norwegen, Serbien, Schweiz (Hertie School of Governance GmbH, 2019a).

${ }^{12}$ Gegenstand des Projekts sind dabei Vergaben oberhalb und unterhalb der nationalen Schwellenwerte, die zum Stichtag August 2015 online und frei verfügbar waren. Für den Umfang der deutschen Daten wurden nur TED-Daten berücksichtigt und damit lediglich Oberschwellenwerte. Diese betrafen die Auftragsbekanntmachung, die Vergabebekanntmachung und Details zum Vertrag (Cingolani et al., 2016, S. 10 ff.; Hrubý et al., 2016, S. 4). ${ }^{13}$ Für Deutschland ist dies https://opentender.eu/de/.
} 
(Hertie School of Governance GmbH, 2019a). In Abschnitt 7.2.1.3 wird hierauf im Zusammenhang mit der Erläuterung von Vorgehensmodellen nochmals eingegangen.

Ebenfalls kommen in Abschnitt 7.2. bei der Erörterung der organisatorischen Umsetzung offener öffentlicher Einkaufsdaten nochmals die Projekte „FragDenStaat“ und das „Transparenzranking“ zur Sprache. Die Projekte rund um „Open Budgets“ und „Offener Haushalt“ zeigen, dass öffentliche Einkaufsdaten auch im Kontext der Haushalts- und Finanzdaten verortet werden können (von Lucke et al., 2012, S. 4). Auf sie wird hier nur verwiesen.

\subsubsection{Open Government Partnership}

Die Open Government Partnership (OGP) wurde am 20. September 2011 von der US-amerikanischen und der brasilianischen Regierung ins Leben gerufen und befasst sich mit Open Government. ${ }^{14}$ Bis dato sind 79 Länder mit etwa 3100 Absichtserklärungen der OGP beigetreten, weitere Länder sind dabei, ihre Mitgliedschaft in der Initiative vorzubereiten. Das Ziel der Initiative ist die Erhöhung der Transparenz und Rechenschaftspflicht sowie der Ansprechbarkeit der Regierungen. Hierüber soll eine Verbesserung in der Regierungsführung sowie der Services für die Bürger erreicht werden. Die Mitglieder der Initiative haben sich verpflichtet, in ihren Ländern Aktionspläne für mehr Transparenz, Bürgerbeteiligung und Verwaltungsmodernisierung zu erstellen und regelmäßig begutachten zu lassen (Open Government Partnership, 2019a).

Die OGP befasst sich in einem ihrer Schwerpunktthemen mit Antikorruption. Dabei fokussiert sie auf zwei Themen, die aus Sicht der Organisation den größten Einfluss haben: Die Erstellung und die Nutzung von öffentlichen Eigentumsregistern und Transparenz über öffentliche Verträge. Laut OGP verausgaben die weltweiten Regierungen geschätzt 9,5 Billiarden US-Dollar jährlich durch öffentliche Verträge. Allerdings sind die Vertragsinformationen der Öffentlichkeit in den meisten Ländern nicht zugänglich. Entsprechend stellen öffentliche Verträge ein großes Risiko für Korruption im öffentlichen Sektor dar. Bis 2018 haben 23 Länder und vier lokale Regierungen erklärt, ihren öffentlichen Vertrags-

\footnotetext{
${ }^{14}$ Die Gründungsländer waren Brasilien, Indonesien, Mexiko, Norwegen, Philippinen, Südafrika, UK und USA (Open Government Partnership, 2019a). Finanziert wird die Initiative durch Beiträge der Mitgliedsstaaten (abhängig von der Wirtschaftskraft des Landes), Hilfsorganisationen sowie private Stiftungen. Das jährliche Budget beläuft sich auf etwa 10 Millionen US-Dollar (Open Government Partnership, 2019c).
} 
erstellungsprozess zu öffnen. Deutschland zählt nicht dazu (Open Government Partnership, 2019b).

Die Bundesrepublik gab im Dezember 2016 die Teilnahme an der OGP bekannt. Dies stellt laut Bundesministerium des Innern, für Bau und Heimat (BMI) zusammen mit dem 2017 in Kraft getretenen Open Data-Gesetz (siehe Abschnitt 7.1.4) einen deutlichen Schritt zu mehr Transparenz, mehr Teilhabe und Stärkung der Innovationsfähigkeit Deutschlands dar (Bundesministerium des Innern, 2016). Im August 2017 wurde der erste, einen Zeitraum von zwei Jahren abdeckende „Nationale Aktionsplan“ (NAP) eingereicht. Er umfasst 15 Verpflichtungen mehrerer Bundesressorts. Hierunter fällt kein Projekt zur Stärkung der Transparenz öffentlicher Verträge (ein Hauptpfeiler der OGP zur Antikorruption). Hingegen finden sich unter den Verpflichtungen die Schaffung von Rahmenbedingungen für die OGP-Teilnahme, die Umsetzung von Open Data in die Verwaltungspraxis und die Förderung des Open Data-Umfelds (Bundesministerium des Innern, 2017b).

Der zweite NAP im Jahr 2019 forderte erstmalig unter der Nummer 36 eine Öffnung der öffentlichen Einkaufsdaten (Open Government Partnership Deutschland e. V., 2019, S. 16 f.). Es seien „Open Data für Planung, Ausschreibung und Vergabeverfahren [zu] implementieren“, und zwar ohne Trennung in Oberund Unterschwellenbereich. Es wurde empfohlen, die Bereitstellung über das Portal GovData und mithilfe des OCDS umzusetzen. Die regierungsinterne Abstimmung startete Ende Mai 2019 (Open Government Partnership Deutschland e. V., 2019, S. 16 f.). Der Vorschlag wurde sodann im August 2019 abgelehnt. Die Bundesregierung begründete die Ablehnung im Wesentlichen damit, dass die Vergabestatistik ausreichend Transparenz schaffe und das im Aufbau befindliche Wettbewerbsregister bereits ein einzigartiger Vorstoß zur Korruptionsprävention sei (Bundeskanzleramt, 2019c, S. 5). Die Argumentation macht deutlich, dass der Umfang der Forderungen entweder nicht verstanden oder bewusst ignoriert wurde. So ist beispielsweise im Unterschwellenbereich die Transparenz weiterhin unzureichend, ungeachtet der Vergabestatistik (die in Abschnitt 6.2.2 noch genauer erläutert wird).

Seit 01. Oktober 2019 ist Deutschland nun eines von 22 Mitgliedern des Lenkungsausschusses (Braun, 2019; Bundeskanzleramt, 2019b). Möglicherweise kann über diese Vorbildrolle die Öffnung des öffentlichen Einkaufs gezielter vorangetrieben werden. 


\subsubsection{Open Contracting Partnership}

Die Open Contracting Partnership (OCP) wurde 2012 gegründet und stand bis 2014 unter der Ägide der Weltbank. Dann wurde sie in ein unabhängiges Programm überführt. Das gesamte verfügbare Einkommen der Organisation belief sich 2017 auf 4,16 Millionen US-Dollar (Open Contracting Partnership, 2019c). ${ }^{15}$

Die Idee zur OCP geht auf motivierte Politikexperten und Aktivisten zurück, die glauben, dass bessere offene Daten und ein stärkeres Engagement einer Gruppe Gleichgesinnter öffentliche Projekte, Produkte und Dienstleistungen verbessern können. Entsprechend fasst die Initiative ihre Absicht zusammen: „We open up public contracting through disclosure, data and engagement so that the huge sums of money involved are spent honestly, fairly, and effectively“ (Open Contracting Partnership, 2019a). Die OCP setzt sich für einen Kulturwandel ein, der auch den Wandel von geschlossenen zu offenen Verträgen einschließt (,open by default“). Sie unterstuitzt ein breites Netzwerk an Partnern, die Projekte zur Öffnung des Einkaufs nach vorn bringen sowie den OCDS anwenden, und sie fördert gemeinsames Lernen sowie Best Practices und Transparenz.

Die OCP beschreibt sich selbst als eine Initiative vieler unterschiedlicher Akteure („Multi-Stakeholder-Initiative“), die weltweit mit Partnern in der Regierung, in Unternehmen, der Zivilgesellschaft, im akademischen Bereich, den Medien und mit der lokalen Ebene zusammenarbeitet, um die öffentlichen Einkaufsdaten zu öffnen und so Probleme zu lösen (Open Contracting Partnership, 2019a). Das Kernprodukt der Initiative ist der im Jahr 2014 eingeführte „Open Contracting Data Standard“ (OCDS). Er beschreibt, wie öffentliche Einkaufsdaten strukturiert über den gesamten Einkaufsprozess von der Planung bis zur Implementierung veröffentlicht werden können (Open Contracting Partnership, 2019g). Des Weiteren bietet die Initiative umfangreiches Lernmaterial ${ }^{16}$ sowie Umsetzungsbeispiele an. Sie bietet eine gute operative Umsetzungsbegleitung beim Öffnen der Einkaufsdaten (zum Vorgehen siehe Abschnitt 7.2.1.2). 18 Länder wenden den OCDS bereits an; Deutschland gehört nicht dazu (Open Contracting Partnership, 2019j).

\footnotetext{
${ }^{15}$ Finanziert wird die OCP über das Budget der Stadt New York sowie weiterer Institutionen, zum Beispiel 2017: Development Gateway, European Bank for Reconstruction and Development (EBRD), Department for International Development (DFID), Hivos, Omidyar Network, Open Society Foundation und die William and Flora Hewlett Foundation (Kluttz et al., 2017, S. 15).

${ }^{16}$ Bei den Lernmaterialien ist zum Beispiel die Datenbank aller Softwarelösungen zu Open Contracting interessant (siehe https://www.open-contracting.org/data-standard/tools/).
} 


\subsubsection{Contracting Five (C5) Partnership}

Abschließend sei die im Zusammenhang mit der Weltbank schon erwähnte „Contracting Five (C5) Partnership“ skizziert. Hierbei handelt es sich um eine freiwillige Zusammenarbeit einzelner Experten und Befürworter in Regierungen, die für eine Stärkung der Offenheit, Innovationstätigkeit und Integrität, eine verbesserte Wirtschaft sowie erhöhtes zivilgesellschaftliches Engagement im öffentlichen Vertragswesen und Einkauf stehen (Contracting Five, 2019d). Sie hat insbesondere zum Gegenstand, die technische Zusammenarbeit in der Umsetzung einer Open Data-Richtlinie für den öffentlichen Einkauf sowie Diskussionen rund um die positiven Effekte dieser Anwendungsbeispiele zu fördern, die Anwendung des OCDS zu unterstützen und einen Fokus auf transparente und offene Steuerung zu legen (Contracting Five, 2019c). Die initialen Mitgliedsstaaten auf Basis des Open Government Partnership's Paris World Summit im Dezember 2016 sind Kolumbien, Frankreich, Mexiko, Großbritannien und die Ukraine. Etwas später kam Argentinien hinzu. Die Finanzierungsstruktur wird nicht näher erläutert (Contracting Five, 2016).

OCP, OGP, Weltbank, EBRD und OECD sind führend mit Blick auf globale Richtlinien und deren Umsetzungen. Sie sind eingeladen, der C5 als inoffizielle Mitglieder und Unterstützer beizutreten. Die OCP und OECD haben bereits organisatorische Unterstützung angeboten (Contracting Five, 2019a).

Im Anhang findet sich eine Tabelle, die eine Gegenüberstellung der vorgestellten IGOs und NGOs und ihres Bezugs zum offenen öffentlichen Einkauf leistet (Tabelle A.3).

\subsection{Die Ziele des offenen öffentlichen Einkaufs}

Auf Grundlage des gewonnenen Überblicks über Regierungsprogramme und Absichten der zivilgesellschaftlichen Organisationen müssen die Ziele des öffentlichen Einkaufs erläutert werden, um die Stärken, Schwächen, Chancen und Risiken, die sich aus der Öffnung des öffentlichen Einkaufs für die wesentlichen Akteure ergeben können, nachvollziehen zu können. Damit soll verdeutlicht werden, welchen Beitrag die Öffnung öffentlicher Einkaufsdaten für die allgemeinen Ziele eines öffentlichen Einkaufs leisten kann.

Die Ziele des öffentlichen Einkaufs lassen sich gut anhand des analytischen Bezugsrahmens für öffentliche Einkaufsaktivitäten von Schapper in Kombination mit den drei Säulen des Leitbilds von Open Government 
(Transparenz, Partizipation und Kollaboration) gemäß Abbildung 5.3 darlegen. Innerhalb des Kreises befindet sich das Zieledreieck des öffentlichen Einkaufs, welches grundsätzliche Ziele (beispielweise das beste Preis-Leistungsverhältnis), Steuerungsaufgaben (zum Beispiel über Regulierung, Wettbewerb und faire Zusammenarbeit), aber auch Rechenschaftspflichten über politische Ziele und volkswirtschaftliche Auswirkungen in Einklang bringen muss.

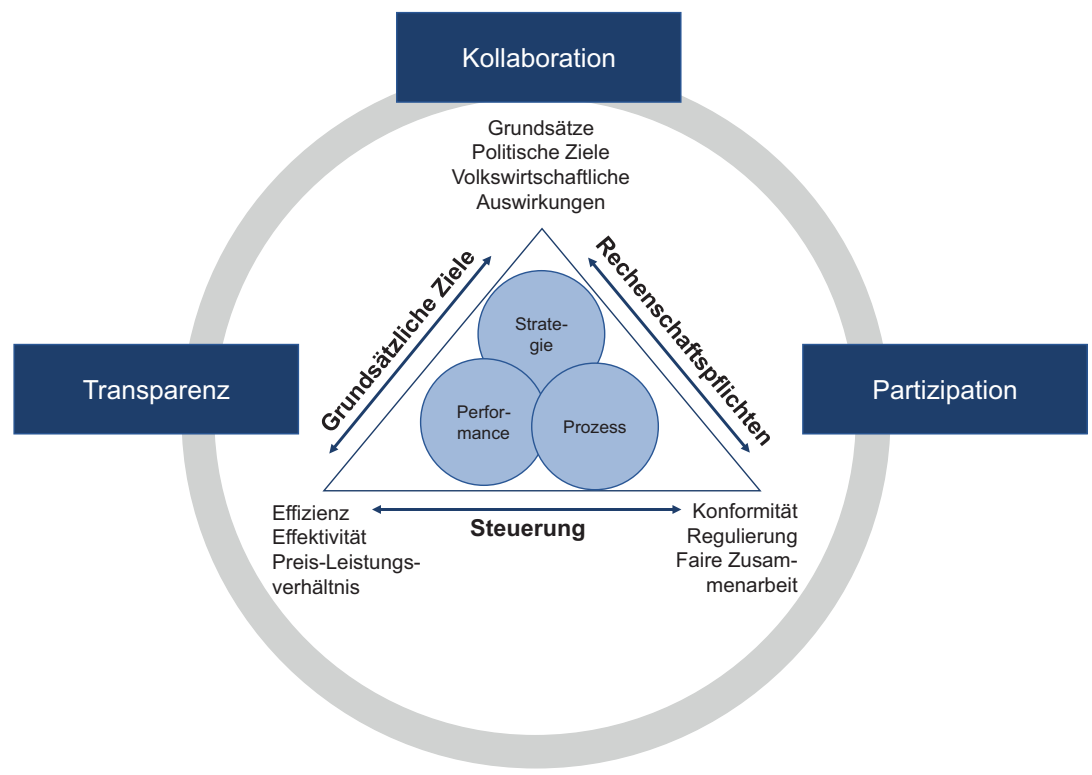

Abbildung 5.3 Bezugsrahmen des offenen öffentlichen Einkaufs (in Anlehnung an Schapper et al., 2006, S. 16, übersetzt durch Verfasserin)

In diesen Bezugsrahmen lassen sich die vielseitigen Unterziele, die zum Beispiel aus den geschilderten politischen Positionen beziehungsweise dem Vergaberecht im Vorgriff auf Abschnitt 6.2.1 abgeleitet werden können, integrieren, wie Tabelle 5.1 illustriert: 
Tabelle 5.1 Beispielhafte Ziele des öffentlichen Einkaufs

\begin{tabular}{|c|c|c|}
\hline $\begin{array}{l}\text { Effizienz, Effektivität, } \\
\text { Preis-/Leistungsver- } \\
\text { hältnis }\end{array}$ & $\begin{array}{l}\text { Grundsätze, politische } \\
\text { Ziele, volkswirtschaft- } \\
\text { liche Auswirkungen }\end{array}$ & $\begin{array}{l}\text { Konformität, } \\
\text { Regulierungen, faire } \\
\text { Zusammenarbeit }\end{array}$ \\
\hline $\begin{array}{l}\text { - Wirtschaftlichkeit } \\
\text { - Sparsamkeit } \\
\text { - Wettbewerb } \\
\text { - Bessere Passfähigkeit, } \\
\text { Auswahl und Qualität } \\
\text { der Produkte und } \\
\text { Dienstleistungen } \\
\text { - Bessere IT-Systeme } \\
\text { - Berücksichtigung von } \\
\text { Innovationen } \\
\text { - Förderung von KMUs }\end{array}$ & $\begin{array}{l}\text { - Einhaltung des Ver- } \\
\text { gaberechts } \\
\text { - Reduktion von Korrup- } \\
\text { tion } \\
\text { - Demokratische/gesell- } \\
\text { schaftliche Teilhabe }\end{array}$ & $\begin{array}{l}\text { - Steuerung (Gover- } \\
\text { nance) des öffentlichen } \\
\text { Einkaufs und der } \\
\text { Digitalisierung } \\
\text { - Rechenschaftspflicht } \\
\text { - Integrität und Glaub- } \\
\text { würdigkeit } \\
\text { - Aufdeckung und } \\
\text { Sanktionierung von } \\
\text { Verstößen } \\
\text { - Entwicklung eines } \\
\text { sozialen und nachhal- } \\
\text { tigen öffentlichen } \\
\text { Einkaufs }\end{array}$ \\
\hline
\end{tabular}

Die Betrachtung einer möglichen Öffnung der öffentlichen Einkaufsdaten lässt es zu, diese Ziele mit den Leitmotiven des offenen Verwaltungs- und Regierungshandelns - Transparenz, Partizipation und Kollaboration - zu kombinieren. Das folgende Kapitel vertieft nun die unterschiedlichen Akteure und die jeweiligen Stärken und Chancen, aber auch Schwächen und Risiken, die sich aus der Öffnung eines öffentlichen Einkaufs für diese Interessengruppen ergeben könnten.

\subsection{Die Akteure des offenen öffentlichen Einkaufs}

Mit dem öffentlichen Einkauf kommen unterschiedliche Akteure in Berührung. Gemäß Abbildung 5.4 können dies beispielsweise sein: Akteure des Marktes (Wirtschaft), des Staates (Verwaltung und Politik), des Querschnitts (Medien und Wissenschaft) sowie der Zivilgesellschaft (Bürger und NGOs). Für sie alle können sich aus einer Öffnung öffentlicher Einkaufsdaten unterschiedliche Potentiale, aber auch Risiken ergeben. 

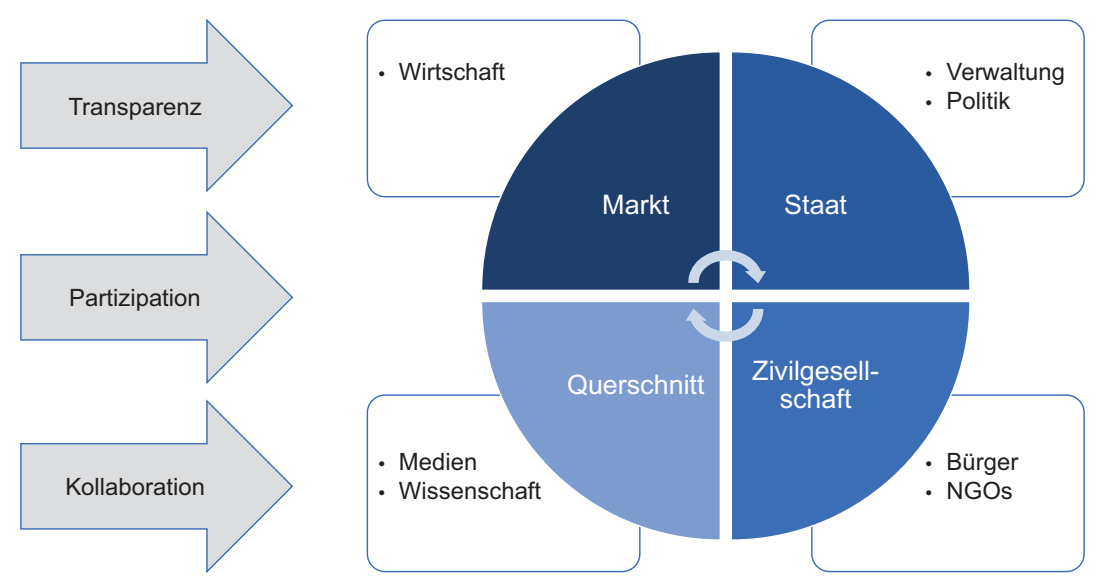

Abbildung 5.4 Akteure des offenen öffentlichen Einkaufs

Die Betrachtung nach einzelnen Gruppierungen und die Aufschlüsselung der Chancen und Risiken ist sinnvoll, da die unterschiedlichen Bedürfnisse der Personengruppen einen differenzierten Umgang mit dem Thema offene öffentliche Daten erfordern.

\section{Vor- und Nachteile der Öffnung des öffentlichen Einkaufs für die Wirtschaft}

Die Akteure der Wirtschaft sind rechtlich selbständige Wirtschaftseinheiten, die Güter oder Dienstleistungen am Markt und damit auch dem öffentlichen Sektor anbieten. In der Bereitstellung öffentlicher Einkaufsdaten ergibt sich für sie vor allem die Chance eines zeit- und kostensparenden Zugangs zu Informationen von Vergabeverfahren und eine größere Teilnahmemöglichkeit. Aktuell bietet nur der ineffiziente Zugang zu verschiedenen Portalen mit teilweise unterschiedlichen Zugangsprozeduren eine breitere Sicht auf Ausschreibungen (siehe Abschnitt 7.3.9.4). Darüber hinaus können Vertreter der Wirtschaft diese Daten aber auch nutzen, um damit selbst Geschäftsmodelle zu entwickeln, zum Beispiel rund um die journalistische Aufbereitung dieser Daten, Bereitstellung von Analyse-Werkzeugen zur gezielten Auswertung der Daten, Software für die Automatisierung der Datenweiterverarbeitung, Erstellung von Berichten oder die Programmierung entsprechender Apps (siehe Abschnitt 7.2.2, Kapitel 8, 9).

Eine mögliche Schwäche könnte sein, dass die Verankerung der Offenlegung öffentlicher Daten in den Verträgen einen neuen Umgang mit dieser ungewohnten Transparenz erfordert, ebenso die Bewertung, was genau als Geschäfts- und Betriebsgeheimnis gilt (siehe Abschnitt 7.1.3). Als Risiko wäre zu benennen, 
dass eventuell unangenehme Tatsachen aufgedeckt werden. Tabelle 5.2 fasst die Aspekte nochmal zusammen.

Tabelle 5.2 SWOT-Analyse offene öffentliche Einkaufsdaten für die Wirtschaft

\section{Stärken}

- Vereinfachter Zugriff auf Vergabedaten und somit Zeit- und Kostenersparnis bei der Recherche

- Möglichkeit der Steigerung der Teilnahme an Vergabeverfahren

- Einbeziehung von Lieferantenexpertise vor und bei der Erstellung der Anforderungen

Chancen

- Verbesserte Verfahrens- und Teilnahmetransparenz

- Wirtschaftsförderung mittels Daten durch z. B. höheren Wettbewerb, auch für KMUs

- Entwicklung neuer Geschäftsmodelle und -möglichkeiten und damit Steigerung der Innovationsfähigkeit rund um den öffentlichen Einkauf

\section{Schwächen}

- Auseinandersetzung damit, dass Vertragsdaten (teilweise) öffentlich gemacht werden

- Neubewertung von Geschäfts- und Betriebsgeheimnissen gegenüber dem Interesse der Allgemeinheit

\section{Risiken}

- Gegebenenfalls Bekanntwerden unangenehmer Tatsachen (z. B. Schlechtleistung) und Erlernen eines angemessenen Umgangs hiermit

- Notwendigkeit zur Veränderung bestehender Geschäftsmodelle, z. B. bei Anbietern von Vergabe- und Bekanntmachungsportalen

\section{Vor- und Nachteile der Öffnung des öffentlichen Einkaufs für die Politik}

In der Politik geht es zumeist um komplexe Sachverhalte, für die eine gut aufbereitete Informationsgrundlage essentiell ist. In der Öffnung der öffentlichen Einkaufsdaten besteht eine große Chance, dass Bewertungsgrundlagen bereitstehen, um fundierte und steueroptimierte Entscheidungen im Sinne der Wähler treffen zu können. Insbesondere kann hiermit ein Beitrag zur Senkung der gesellschaftlichen Kosten geleistet werden, welche durch Korruption entstehen (siehe Abschnitt 6.5.1). Über die transparente Bereitstellung von Verträgen können Wähler im Sinne der Beteiligung hierauf einwirken beziehungsweise diese Entscheidungen besser nachvollziehen. Glaubwürdigkeit und Akzeptanz können gesteigert oder Fehlentscheidungen schneller revidiert und/oder mit Konsequenzen belegt werden.

Voraussetzung ist ein politischer Wille zur Offenlegung, dem die Sorge vor Bekanntwerden korrupter Fälle sowie der Verlust an Glaubwürdigkeit im politischen Handeln gegenübersteht. Das größte Risiko liegt im Ausbleiben sichtbarer Konsequenzen bei Schlechtleistung von Unternehmen oder auch Fehlallokationen der Haushaltsmittel. Tabelle 5.3 fasst die Aspekte nochmals zusammen. 
Tabelle 5.3 SWOT-Analyse offene öffentliche Einkaufsdaten für die Politik

\begin{tabular}{l|l}
\hline Stärken & Schwächen \\
\hline - Beitrag zur Korruptionsbekämpfung und & - Erfordernis des politischen Willens zur \\
somit Senkung der hierdurch entstehen- & Transparenz \\
den gesellschaftlichen Kosten & - Sorge vor Bekanntwerden korrupter Fälle \\
- Beitrag zur Wettbewerbsförderung & und Notwendigkeit einer angemessenen \\
- Erhöhung der Haushaltstransparenz & Auseinandersetzung hiermit \\
\hline Chancen & Risiken \\
\hline - Verbesserte Informations- und Entschei- & - Imageverlust der Politik bei unvorteilhaft \\
dungsgrundlagen & geschlossenen Verträgen oder Korruption \\
- Optimierte Allokation der Haushalts- & - Ausbleiben sichtbarer Konsequenzen bei \\
mittel durch Einbeziehung Dritter bzw. & Fehlallokationen \\
der Bürger in den Entscheidungsprozess & \\
- Verbesserte Nachvollziehbarkeit und & \\
$\begin{array}{l}\text { Glaubwürdigkeit der Entscheidungen } \\
\text { - Schnellere Korrektur falscher Entschei- } \\
\text { dungen }\end{array}$ &
\end{tabular}

\section{Die Nutzung offener öffentlicher Einkaufsdaten für die Verwaltung}

Die Verwaltung agiert eng an der Schnittstelle zum politischen Handeln. Ihre Kernaufgaben sind der Vollzug von Gesetzen und Vorschriften, die Erbringung diverser Dienstleistungen, die Bearbeitung politischer Themen und die Selbstgestaltung der Organisation (Klessmann et al., 2012, S. 63). Diese betreffen auch alle Aufgaben rund um den öffentlichen Einkauf. In diesem Spektrum erzeugt die Verwaltung sehr viele Daten, von denen sie aber gleichzeitig auch profitieren kann, zum Beispiel nach außen gerichtet, wenn es darum geht, bessere Preise zu verhandeln, Schlechtleistungen von Lieferanten schneller zu bewerten oder zusätzliche Unternehmen in den öffentlichen Einkaufsprozess einzubinden oder nach innen gerichtet, um Transparenz über interne Abläufe zu gewinnen und diese zu optimieren. Über eine verbesserte Datenqualität, einen verbesserten Datenaustausch oder die Verknüpfung mit anderen Datenbeständen (siehe Abschnitt 7.3.2.2) könnten so Synergien über Verwaltungseinheiten hinweg gehoben werden, da die neu gewonnene Transparenz den Wissensaustausch erleichtert und Vergleiche zulässt. Weitere Chancen betreffen eine frühzeitige Risikoabwendung durch die Einbindung Dritter (zum Beispiel der Öffentlichkeit) sowie effizientere Nachprüfungsmöglichkeiten für die Rechnungshöfe. Denkbar ist auch eine Verlagerung von Aufgaben der Verwaltung durch die Bereitstellung dieser Daten auf Dritte und somit eine Entlastung.

Jedoch muss der Nutzen der Datenbereitstellung mit ihren Kosten verglichen werden: Nicht alle Datenbestände sind ad hoc maschinenlesbar und in hoher 
Qualität verfügbar. Dies kann anfängliche Investitionen erfordern. Der erforderliche Kulturwandel durch die Erweiterung der Deutungshoheit der Daten auf die Öffentlichkeit darf ebenso wenig unterschätzt werden wie die nötigen Qualifizierungen der Mitarbeiter. Risiken können im Desinteresse oder Unwissen im Umgang mit den Daten liegen (Fehlinterpretationen, Nichtanwendung) sowie in Unsicherheit in der Abwägung der Bewertung von Geschäfts- und Betriebsgeheimnissen. Tabelle 5.4 fasst die Ergebnisse zusammen.

Tabelle 5.4 SWOT-Analyse offene öffentliche Einkaufsdaten für die Verwaltung

\section{Stärken}

- Transparenz im Vergabeverfahren bei gleichzeitiger öffentlicher Kontrolle

- Bessere Einkaufslösungen und Preise

- Beitrag zur Korruptionsbekämpfung

- Erhöhung der Haushaltstransparenz

- Verbesserte Auswertungsmöglichkeiten, insbesondere mit Blick auf die Verknüpfung mit anderen Datenbeständen (Linked Open Data)

\section{Chancen}

- Transparenz über interne Abläufe, Nutzung von Synergieeffekten, Erzielung von Einsparungen

- Erhöhte und vereinfachte Nachprüfungsmöglichkeiten (z. B. durch Rechnungshöfe)

- Über Hinweisgeber frühzeitige Risikoabwendung

- Qualitätssteigerung durch Nutzung von Experten

- Entlastung von Aufgaben durch „Customer Self Service“

- Image- und Akzeptanzgewinn

\section{Schwächen}

- Kulturwandel für die Verwaltung (z. B. Verlust der Deutungshoheit; Umgang mit ,Fehlern')

- Nutzen versus Kosten der Öffnung der Datenbestände

- Mitarbeiterqualifikationen ggf. unzureichend

- Unzureichende Nutzung der Daten durch die Verwaltung selbst

\section{Risiken}

- Desinteresse der Verwaltung und Öffentlichkeit am Angebot offener öffentlicher Einkaufsdaten

- Arbeitsaufwand in der Pflege, Bewertung und Bereitstellung der Daten

- Abwägung öffentliches Interesse versus Geschäfts- und Betriebsgeheimnisse

- Risiko von Fehlinterpretationen

- Zurückhaltung von Daten

\section{Die Bereitstellung öffentlicher Einkaufsdaten für die Bürger}

Bürger und NGOs sind in dieser Arbeit unter dem Oberbegriff der Zivilgesellschaft zusammengefasst. Ein Synonym für Zivilgesellschaft ist oftmals „Bürgergesellschaft“. Allgemein wird unter dem Begriff meist der Teil der Gesellschaft verstanden, der nicht durch den Staat und seine Organe (Behörden, Verwaltungen) gesteuert und organisiert wird. Bürger sind Privatpersonen, die vorrangig im lokal-kommunalen Bereich agieren und von den dortigen 
Ereignissen am ehesten betroffen sind. Mit Blick auf öffentliche Einkaufsdaten ist für die Bürger zum Beispiel die angemessene und nachvollziehbare Verwendung der von ihnen erwirtschafteten Steuermittel relevant, insbesondere im eigenen Wohn- und Arbeitsumfeld. Aufgrund von Informations- und Freiheitsgesetzen haben Bürger einen Anspruch auf Informationen; teilweise ist dies noch eine Holschuld, einige Gesetze betonen aber bereits die aktive Bereitstellungspflicht und eine Bringschuld gegenüber dem Bürger - von ,,open by demand to open by default" (siehe Abschnitt 7.1).

Öffentliche Einkaufsdaten müssen in einer leicht verständlichen Form angeboten werden, da sonst Desinteresse an diesen Daten und einer Mitgestaltung entstehen kann. Die Einbindung der Bürger ist wichtig, um zu erfahren, welche Daten eine Relevanz für sie besitzen, zugleich ist es unabdingbar, dieses komplexe Thema verständlich aufzubereiten, damit der Umgang mit den Auswertungen der Daten angemessen erfolgen kann. Die Risiken liegen neben einem ungleichen Zugang zu den Daten im Ausbleiben nachvollziehbarer Konsequenzen bei ungeeigneter Mittelverwendung. Ein weiteres Risiko besteht darin, dass die Öffnung der öffentlichen Einkaufsdaten nur eine Alibifunktion erfüllt und Verträge oder Vertragsdaten mit dem Argument der Betriebs- und Geschäftsgeheimnisse entweder umfassend geschwärzt oder vollständig zurückgehalten werden. Die Ergebnisse werden in Tabelle 5.5 zusammengefasst.

Tabelle 5.5 SWOT-Analyse offene öffentliche Einkaufsdaten für die Bürger

\section{Stärken}

- Öffentliche Einsicht in alle Verträge

- Überprüfung der Angemessenheit der Verwendung der Steuermittel

\section{Schwächen}

- Bürger müssen informiert und geschult werden, um die Datenangebote bewerten und nutzen zu können

- Mindestens zu Beginn Notwendigkeit der Bewerbung des Datenangebots zur Erhöhung der Nutzung im komplexen Fachgebiet des öffentlichen Einkaufs

\section{Chancen}

\section{Risiken}

- Unterstützung für engagierte Bürger/Vertreter der Zivilgesellschaft

- Proaktive Bereitstellung von Informationen als Grundlage für die politische Mitgestaltung

- Ausbleiben sichtbarer Konsequenzen bei Bekanntwerden von Schlechtleistung/ Fehlallokation von Haushaltsmitteln

- Ungleicher Zugang zu den Daten (unzureichende Barrierefreiheit)

- Beitrag zu einer ,stabileren“ Gesellschaft, indem demokratiegefährdende Elemente wie Korruption weiter reduziert werden

- Optimierte Allokation der Haushaltsmittel durch Einbeziehung der Bürger in den Entscheidungsprozess
- Betriebs- und Geschäftsgeheimnisse werden über das Interesse der Gemeinschaft gestellt

- Fehlinterpretationen aufgrund begrenzten Wissens 


\section{Das Potential der Öffnung öffentlicher Einkaufsdaten für Nichtregierungs- organisationen}

Die Öffnung öffentlicher Einkaufsdaten bietet ein großes Potential zur Umsetzung des Hauptziels von NGOs, der Erzeugung von Transparenz zur Korruptionsbekämpfung. Überdies bietet die Öffnung NGOs die Chance, sich in einer Expertenrolle zwischen Politik, Verwaltung, Wirtschaft und Bürger am Vergabeprozess zu beteiligen, so in der Phase der Planung oder der Ausführung (zum Beispiel Monitoring von Ergebnissen), sozusagen als Intermediär. Hier muss allerdings gewährleistet werden, dass Mitwirkende im Thema des öffentlichen Einkaufs entsprechend geschult werden, damit Daten angemessen ausgewertet werden können. Ebenso würde dies eine Weiterentwicklung der NGOs in Richtung eines Qualitätssicherers bedeuten. Tabelle 5.6 stellt die Ergebnisse dar.

Tabelle 5.6 SWOT-Analyse offene öffentliche Einkaufsdaten für die NGOs

\begin{tabular}{l|l}
\hline Stärken & Schwächen \\
\hline $\begin{array}{l}\text { - Transparenz im Vergabeverfahren bei } \\
\text { gleichzeitiger öffentlicher Kontrolle als } \\
\text { Beitrag zur Korruptionsbekämpfung }\end{array}$ & $\begin{array}{l}\text { Mitwirkende müssen geschult werden, } \\
\text { die Datenangebote entsprechend bewer- } \\
\text { ten und nutzen zu können } \\
\text { - Aufbau von Fachexpertise rund um den } \\
\text { öffentlichen Einkauf }\end{array}$ \\
\hline Chancen & Risiken \\
\hline $\begin{array}{l}\text { - Unterstützung der Organisation in der } \\
\text { Verfolgung ihrer Ziele }\end{array}$ & $\begin{array}{l}\text { Fehlinterpretationen aufgrund begrenzten } \\
\text { Wissens }\end{array}$ \\
$\begin{array}{l}\text { Nutzung der Daten zur Aufklärung spezi- } \\
\text { fischer Sachverhalte }\end{array}$ & $\begin{array}{l}\text { Veränderung der Rolle der Organisation } \\
\text { - Einbindung als Experten im öffentlichen Richtung Qualitätssicherer } \\
\text { Vergabeprozess als Vertreter der Zivil- } \\
\text { gesellschaft }\end{array}$ \\
\hline
\end{tabular}

\section{Der erweiterte Datenzugriff für die Medien}

Medien und Wissenschaft werden der Zielgruppe des Querschnitts zugeordnet, da sie für die Gesellschaft übergreifende Aufgaben wahrnehmen. Das vorrangige Ziel der Medien ist die Herstellung von Öffentlichkeit, aber auch die Kontrolle des Staates. Auch Aktivitäten von Bloggern und Bürgerjournalisten können in diesem Sinne als journalistische Tätigkeiten verstanden werden. Als Vertreter können zum Beispiel Correctiv e. V., Mehr Demokratie e. V. oder netzpolitik. org e. V. genannt werden. Mit dem Zugriff auf öffentliche Verträge bietet sich ihnen die Chance einer überprüfbaren Datengrundlage, was die Aussagefähigkeit der eigenen Arbeit verbessern und helfen kann, Sachverhalte aufzuklären. Gleichzeitig bieten sie auch die Chance für neue Geschäftsmodelle, zum Beispiel als 
Dienstleister von „Datendrehscheiben“ oder in der Entwicklung des Berufsbilds vom klassischen Journalismus hin zum Berufsbild des Datenjournalisten oder -redakteurs (Klessmann et al., 2012, S. 71).

Auch hier liegen mögliche Schwächen in einer zu geringen Fachkenntnis oder unzureichenden Schulungen der Datenjournalisten zum Verständnis und zur Auswertung der Daten. Ein Risiko kann in der Verschiebung der oben erörterten Rollen liegen. Die Argumente werden in Tabelle 5.7 konsolidiert aufgeführt.

Tabelle 5.7 SWOT-Analyse offene öffentliche Einkaufsdaten für die Medien

\begin{tabular}{l|l}
\hline Stärken & Schwächen \\
\hline $\begin{array}{l}\text { - Transparenz im Vergabeverfahren er- } \\
\text { leichtert die Aufklärung spezifischer } \\
\text { Sachverhalte }\end{array}$ & $\begin{array}{l}\text { Ausreichende Schulung der Journalisten } \\
\text { in der Bewertung und Nutzung von } \\
\text { Datenangeboten } \\
\text { - Aufbau von Fachexpertise rund um den } \\
\text { öffentlichen Einkauf }\end{array}$ \\
\hline Chancen & Risiken \\
\hline $\begin{array}{l}\text { - Zusätzliche Datengrundlage für hoch- } \\
\text { wertigere Recherchen und somit bessere }\end{array}$ & $\begin{array}{l}\text { Pehlinterpretationen aufgrund begrenzten } \\
\text { Ausübung der eigenen Kontrollfunktion }\end{array}$ \\
$\begin{array}{ll}\text { - Weiterentwicklung der eigenen Rolle hin } \\
\text { zum Intermediär in der Aufbereitung der } \\
\text { Informationen für den Bürger }\end{array}$ & $\begin{array}{l}\text { Journalismus zum Datenexperten } \\
\text { - Entwicklung weiterer Geschäftsmodelle, } \\
\text { z. B. hin zum Datenjournalismus }\end{array}$ \\
\hline
\end{tabular}

\section{Offene öffentliche Einkaufsdaten als Quelle für die Wissenschaft}

Die Wissenschaft ist ein Oberbegriff für zahlreiche Institutionen, die Erkenntnisgewinn mithilfe wissenschaftlicher Methoden im Bereich der Grundlagenforschung und angewandten Forschung anstreben. Die Stärke der Öffnung öffentlicher Einkaufsdaten liegt darin, dass das Datenmaterial vorläge und nicht gesondert aus einer Vielzahl von Quellen erhoben werden müsste. So könnte die Forschung in diesem Themengebiet unter Reduktion des eigenen Aufwands vorangebracht werden.

Das Risiko liegt allerdings auch in einer gegebenenfalls mangelnden Datenqualität, sodass Ergebnisse verfälscht sein könnten. Eine Öffnung von Daten zieht zudem nach sich, dass die Wissenschaft eher bei der Auswertung denn bereits bei Erhebung der Daten ansetzt, was mit einer Veränderung des Selbstverständnisses verbunden wäre. Tabelle 5.8 zeigt die Zusammenstellung der Ergebnisse. 
Tabelle 5.8 SWOT-Analyse offene öffentliche Einkaufsdaten für die Wissenschaft

\begin{tabular}{|c|c|}
\hline Stärken & Schwächen \\
\hline $\begin{array}{l}\text { - Beitrag zur Wissensgesellschaft und } \\
\text { Partizipation in einem mäßig erforschten } \\
\text { Bereich }\end{array}$ & $\begin{array}{l}\text { - Bezug externer Daten anstelle Erzeugung } \\
\text { eigener Datengrundlagen } \\
\text { - Wandel in der Rolle der Wissenschaft, da } \\
\text { der exklusive Zugang zu Informationen } \\
\text { reduziert wird }\end{array}$ \\
\hline Chancen & Risiken \\
\hline $\begin{array}{l}\text { - Vertiefung und Verbesserung wissen- } \\
\text { schaftlicher Erkenntnisse rund um den } \\
\text { öffentlichen Einkauf } \\
\text { - Nutzung einheitlicher bzw. verifizierter } \\
\text { Datenquellen, bessere Überprüfbarkeit } \\
\text { - Reduktion von Forschungsaufwänden }\end{array}$ & $\begin{array}{l}\text { - Mangelnde Datenqualität } \\
\text { - Fehlinterpretation der Daten }\end{array}$ \\
\hline
\end{tabular}

\subsection{Zwischenfazit: Trotz wirtschaftlicher Relevanz kein konzertierter Fokus auf Öffnung des öffentlichen Einkaufs}

Die Darstellung der Begriffe, Haltungen, Ziele und Akteure, die im Zusammenhang mit öffentlichem Einkauf eine Rolle spielen, konnte einige wichtige Aspekte klären. Zunächst erwies sich, dass auf der Seite der internationalen und europäischen Regierungsprogramme die Öffnung der Verwaltungsdaten allgemein im Fokus steht und nicht die Förderung einzelner Datenkategorien. Die Ausrichtung der Einkaufsoptimierung orientiert sich somit überwiegend an der strukturellen und technischen Optimierung in Bezug auf gemeinsame Infrastrukturen, weitere Standardisierung, die transaktionale Prozessierung und eine stärkere ebenenübergreifende Zusammenarbeit der Behörden. Eine Ausnahme ist die G8-Open Data-Charter, in der öffentliche Einkaufsdaten eindeutig als zu öffnende Datenkategorie benannt werden. Die Forderungen nach Öffnung der öffentlichen Einkaufsdaten werden insbesondere durch NGOs artikuliert, in denen Deutschland nach und nach einen aktiveren Part übernimmt, auch wenn dies, gemessen an anderen Mitgliedsstaaten wie zum Beispiel Frankreich, eher zögerlich geschieht.

Sichtlich kann die Offenlegung der öffentlichen Einkaufsdaten eine Vielzahl der Ziele des öffentlichen Einkaufs fördern, in allererster Linie die Unterstützung von Transparenz und Integrität, die dann idealerweise zur Reduktion von Korruption, einer effizienteren Mittelverwendung und einer höheren politischen 
Glaubwürdigkeit führt. Hier gehen die Ausführungen mit von Lucke konform, der konstatiert: „Der transparente Umgang mit Zuschlägen, Verträgen, Subventionszahlungen und Entwicklungshilfe [...] trägt zur Haushaltstransparenz, zur öffentlichen Kontrolle und zur Korruptionsbekämpfung bei“ (von Lucke et al., 2012, S. 32).

Die Potentiale der Transparenz und Erhöhung der Integrität bestehen für alle Akteure. Zugleich bietet die Offenlegung der öffentlichen Einkaufsdaten für die einzelnen Akteure neben Stärken und Chancen auch Schwächen und Risiken. Ohne die Vor- und Nachteile fundiert im Einzelnen abwägen zu können, lässt sich feststellen: Über alle Akteure hinweg gibt es eine Indikation, dass eine Öffnung der Einkaufsdaten sinnvoll wäre, da Risiken und Schwächen bearbeitbar erscheinen.

Gleichwohl fehlt ein konzentrierter und konzertierter Fokus darauf, eine solche Öffnung umzusetzen. Insgesamt haben die internationalen und europäischen Regierungsprogramme zwar die Notwendigkeit sowohl der Öffnung der Verwaltungsdaten als auch der Digitalisierung als Treiber einer modernen Verwaltung anerkannt. Doch steht die Öffnung der öffentlichen Einkaufsdaten nicht ausdrücklich auf der Agenda. Deutschland ist der OCP bislang nicht beigetreten, doch könnte der Beitritt dazu führen, dass das Thema offene Einkaufsdaten in Zusammenarbeit mit der OKFN und OGP frühzeitig und gesamthaft in seiner Anwendung auf Deutschland vorbereitet werden kann. Als vorteilhaft könnte sich Deutschlands Rolle im Lenkungsausschuss der OGP erweisen.

Die Ausführungen haben auch gezeigt, dass Akteure vieler verschiedener Gruppen auf diversen Ebenen von dem Thema betroffen sind und entsprechend einbezogen werden müssten. Die Berücksichtigung verschiedener Perspektiven scheint notwendig, um die abstrakte Forderung nach Offenlegung der öffentlichen Einkaufsdaten mit konkreten Maßnahmen zu verbinden, die Chancen auszuarbeiten, die Risiken nachzuvollziehen und zu minimieren.

Für die vorliegende Untersuchung ergibt sich aus den bisherigen Erkenntnissen, dass die beiden Faktoren öffentlicher Einkauf und offene öffentliche Einkaufsdaten zunächst einmal genauer betrachtet werden müssen, um auf dieser Grundlage empirisch zu hinterfragen, inwiefern sich konkret für diesen Bereich die Potentiale und Risiken einer Öffnung der öffentlichen Beschaffung darstellen. Im folgenden Kapitel wird daher die Ausgangssituation des öffentlichen Einkaufs in den für diese Arbeit relevanten Themen dargelegt. 
Open Access Dieses Kapitel wird unter der Creative Commons Namensnennung 4.0 International Lizenz (http://creativecommons.org/licenses/by/4.0/deed.de) veröffentlicht, welche die Nutzung, Vervielfältigung, Bearbeitung, Verbreitung und Wiedergabe in jeglichem Medium und Format erlaubt, sofern Sie den/die ursprünglichen Autor(en) und die Quelle ordnungsgemäß nennen, einen Link zur Creative Commons Lizenz beifügen und angeben, ob Änderungen vorgenommen wurden.

Die in diesem Kapitel enthaltenen Bilder und sonstiges Drittmaterial unterliegen ebenfalls der genannten Creative Commons Lizenz, sofern sich aus der Abbildungslegende nichts anderes ergibt. Sofern das betreffende Material nicht unter der genannten Creative Commons Lizenz steht und die betreffende Handlung nicht nach gesetzlichen Vorschriften erlaubt ist, ist für die oben aufgeführten Weiterverwendungen des Materials die Einwilligung des jeweiligen Rechteinhabers einzuholen.

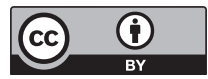

